\section{Aktuelle Vorstellungen zur Entstehung von Komedonen (Komedogenese) und Implikationen für eine Pathogenese-orientierte Aknetherapie}

\author{
Recent Understandings of the Development of Comedones (Comedogenesis) and \\ Implications for Pathogenesis-Related Acne Therapy
}

\section{Zusammenfassung}

Für die Entstehung von Komedonen bei der Akne werden verschiedene pathogenetische Faktoren verantwortlich gemacht. $\mathrm{Zu}$ den endogenen Faktoren zählen P. acnes, Interleukin-1 $\alpha$, androgene Effekte auf die Follikelkeratinozyten, Veränderungen des Retinol- oder Dehydroretinolgehaltes der Talgdrüse, ein relativer Linolsäuremangel im Follikel sowie komedogene Bestandteile des Talges. Exogene Faktoren wie komedogene Substanzen in Pflegemitteln und Kosmetika tragen zur Komedogenese bei. Das Zusammenspiel endogener und exogener Faktoren führt zu einer gestörten Verhornung (Proliferations-Retentions-Hyperkeratose) im Infrainfundibulum des Follikelausführungsganges. Eine Pathogenese-orientierte Aknetherapie ist auf die Normalisierung der epithelialen Verhornung mit Beseitigung der Komedonen und Verhinderung ihrer Neubildung ausgerichtet.

\section{T. Jansen \\ J. Dissemond \\ S. Grabbe}

Abstract

A variety of pathogenetic factors may be responsible for the development of comedones in acne. Endogenic factors include $P$. acnes, interleukin- $1 \alpha$, androgenic effects on follicular keratinocytes, changes of the retinol or dehydroretinol content of the sebaceous gland, a relative linoleic acid deficiency within the follicle, and comedogenic sebum components. Exogenic factors such as comedogenic ingredients of skin care products and cosmetics contribute to comedogenesis. Endogenic and exogenic factors lead to disturbed keratinization (proliferation-retention hyperkeratosis) of the follicular infrainfundibulum. Pathogenesis-related acne therapy will normalize epithelial keratinization with clearing and prevention of comedones.

\section{Einleitung}

Die Akne ist eine an den Talgdrüsenfollikel gebundene Hauterkrankung mit vorwiegender Manifestation in der Pubertät. Das klinische Erscheinungsbild ist polymorph mit primären (nichtentzündlich: geschlossene und offene Komedonen), sekundären (entzündlich: Papeln, Pusteln, Knoten) und tertiären (postentzündlich: Narben) Effloreszenzen. Bevorzugt sind das Gesicht und der V-förmige Brust- und Rückenabschnitt befallen.
Der Altersgipfel der Kometonenakne liegt um das 12. Lebensjahr, der Gipfel der entzündlich-papulösen Akne um das 16. Lebensjahr, während die pustulöse und knotige Akne gewöhnlich zwischen dem 16. und 20. Lebensjahr auftreten. Im typischen Fall bildet sich die Akne mit Beginn des dritten Lebensjahrzehntes zurück, jedoch persistiert die Erkrankung in einem Teil der Fälle oder tritt erst nach der Pubertät in Erscheinung.

Die endogene, in der Pubertät einsetzende Akne ist eine multifaktorielle Erkrankung, deren Ausprägung durch die Summe der einzelnen pathogenetischen Faktoren bestimmt wird [22,34,42]. 
Dazu zählen genetische Prädisposition, Hormone (Androgene), Talgüberproduktion (Seborrhö), follikuläre Hyperkeratose (Komedogenese), mikrobielle Hyperkolonisation (Propionibacterium acnes, $P$. acnes) sowie Entzündung und Immunreaktion. In den letzten Jahren haben neue Erkenntnisse zu einem besseren Verständnis der komplexen Pathogenese der Akne geführt.

\section{Komedogenese: Erscheinungsformen und Ursachen}

Die primäre Veränderung der Akne besteht in einer gestörten Verhornung in den Ausführungsgängen (Infundibula) der Talgdrüsenfollikel $[25,30,43,61]$. Im tiefer gelegenen Abschnitt des Infundibulums, dem Infrainfundibulum, werden mehr Keratinozyten als normal gebildet. Sie differenzieren sich zu Hornzellen, die miteinander verkleben und so zusammenhängende Hornlamellen bilden, die zunehmend das Lumen des Follikelkanals verengen. Unter physiologischen Bedingungen werden die in den Follikelinfundibula gebildeten und in das Lumen abschilfernden Hornzellen kontinuierlich durch den höher gelegenen Abschnitt des Infundibulums, das Akroinfundibulum, an die Hautoberfläche abgegeben. Die ersten histologischen und ultrastrukturellen Veränderungen mit Vermehrung von Hornzellmaterial im Follikelkanal sind bei aknedisponierten Individuen bereits vor Einsetzen der Pubertät nachweisbar.

In immunhistochemischen und autoradiographischen Untersuchungen ließ sich zeigen, dass eine Hyperproliferation der Keratinozyten des Follikelepithels für die Ansammlung des Hornzellmaterials im Follikelkanal verantwortlich ist. Bei Verwendung des monoklonalen Antikörpers Ki-67, der von aktiv sich teilenden Zellen exprimiert wird, zeigten basale Keratinozyten in Komedonen von Aknepatienten eine stärkere Anfärbung als basale Keratinozyten in klinisch nicht veränderten Follikeln von Hautgesunden [27]. Die Hyperproliferation der Follikelkeratinozyten ließ sich auch durch eine verstärkte ${ }^{3} \mathrm{H}$-Thymidin-Markierung und einen verstärkten ${ }^{3} \mathrm{H}$-Histidin-Einbau in Komedonen aufzeigen [45]. Mit der in situ-Hybridisierung fand sich bei Aknepatienten eine starke suprabasale Aktivität der Keratine 6 und 16, phänotypischen Markern von hyperproliferierenden und pathologisch differenzierenden Keratinozyten, in Komedonen, dagegen war eine geringe suprabasale Aktivität in klinisch nicht veränderten Follikeln vorhanden [18]. Die vermehrte Keratinexpression in der Komedowand ist als Folge der erhöhten Zellerneuerungsrate in der Haut von Aknepatienten aufzufassen.

In der Umgebung von Komedonen und klinisch nicht veränderten Follikeln von Aknepatienten wurde eine aberrante Expression der Integrine $\alpha_{2}$ und $\alpha_{3}$ nachgewiesen [17]. Integrine sind Adhäsionsmoleküle mit Einfluss auf die Proliferation und Differenzierung der Keratinozyten. Die Liganden für die Integrine $\alpha_{2}$ und $\alpha_{3}$ (Typ-IV-Kollagen und Laminin) sind Bestandteile der Basalmembranzone. Diese Befunde lassen vermuten, dass die aberrante Integrinexpression durch Veränderungen in den extrazellulären Matrixproteinen zustande kommt.

Darüber hinaus zeigten Aknepatienten eine Hyperproliferation der Follikelkeratinozyten in klinisch nicht veränderten Follikeln [27]. Histologische Untersuchungen an Hautproben von Aknepatienten ergaben, dass klinisch nicht veränderte Follikel bereits Merkmale von Mikrokomedonen aufweisen können [1]. Das ist der Grund, warum topisch applizierte Aknetherapeutika nicht auf die sichtbaren Effloreszenzen alleine, sondern auf die gesamte befallene Aknehaut aufgetragen werden müssen.

Auf diese Weise entsteht eine follikuläre Proliferations-Retentions-Hyperkeratose, die zur Ausbildung eines Mikrokomedos, der initialen Läsion der Akne, führt. Ein Mikrokomedo ist nur mikroskopisch, nicht aber mit bloßem Auge erkennbar. Das Follikelepithel wird akanthotisch. Im Lumen der Ausführungsgänge werden Haare retiniert. Die Talgdrüsen sind noch groß. Ein Mikrokomedo benötigt mehrere Wochen, um sich zu einem klinisch erkennbaren, geschlossenen Komedo (engl. „whitehead“) weiter zu entwickeln. Hierbei handelt es sich um ein weißliches, festes, Miliumartiges Knötchen von 1-2 mm Durchmesser, dessen Öffnung sehr klein und mit bloßem Auge gewöhnlich nicht erkennbar ist. In diesem Stadium ist das Follikelepithel oft hyperplastisch und besitzt ein auffälliges Stratum granulosum, obwohl es im weiteren Verlauf mit zunehmender Hornzellansammlung wieder dünner wird. Die Talgdrüsen bilden sich zurück, verschwinden aber nie völlig. Ultrastrukturell fallen intrazelluläre Lipidvakuolen und leer erscheinende, wenig osmiophile Zellen auf. Die zunehmende Ansammlung von sich vorschiebenden, dunkel pigmentierten Hornzellmassen führt zu einer Ausweitung der Follikelöffnung mit Ausbildung eines offenen Komedos (engl. „blackhead“), der einen Durchmesser bis zu 5 mm erreichen kann. Das Pigment der Komedonen, bei offenen Komedonen mit bloßem Auge, bei geschlossenen Komedonen nur mikroskopisch sichtbar, ist weder Schmutz noch oxidiertes Lipidmaterial, sondern Melanin. Offene Komedonen bilden sich spontan zurück oder entwickeln sich zu entzündlichen Akneläsionen weiter.

Da alle Effloreszenzen bei der Acne vulgaris zunächst ein Mikrokomedonenstadium durchlaufen, wird die Notwendigkeit einer konsequenten Schälbehandlung (Keratolyse), die auch die Follikelinfundibula erreicht, verständlich.

Ausgangspunkt für die Entwicklung von entzündlichen Akneläsionen kann jedes Stadium der Komedobildung sein [39]. Häufig verhält sich die Anzahl der offenen Komedonen und der entzündeten Follikel umgekehrt proportional. Am häufigsten tritt die Entzündung bereits im Stadium des Mikrokomedos ein. In diesem Fall sieht es so aus, als bekäme der Patient eine entzündliche Akne, ohne dass er zuvor das Komedostadium durchlaufen hätte. Im Unterschied zum Mikrokomedo und geschlossenen Komedo ist der offene Komedo reaktionsträger und tritt seltener in ein Entzündungsstadium ein.

Es wird angenommen, dass sowohl Haarfollikel als auch Talgdrüsenfollikel einem bestimmten Lebenszyklus folgen. Zyklen normaler Follikel und Komedonenzyklen sollen sich gegenseitig beeinflussen [7]. Zyklen normaler Follikel könnten dann in Komedonenzyklen mit nachfolgender Ausbildung von Akneeffloreszenzen übergehen. Umgekehrt könnten Komedonenzyklen in Zyklen normaler Follikel mit nachfolgender Rückbildung übergehen. Diese Annahme würde erklären, warum sich viele Komedonen auch ohne Therapie zurückbilden.

Die follikuläre Hyperkeratose führt entgegen früherer Annahmen nicht zu einem vollständigen Verschluss der Follikelöffnung. Das Akroinfundibulum nimmt an dem Vorgang gewöhn- 
lich nicht teil. Würde sich im Akroinfundibulum eine Hyperkeratose ausbilden, wäre die Follikelöffnung von Beginn an dilatiert. Die erste klinisch sichtbare Läsion wäre dann ein offener und nicht ein geschlossener Komedo.

Das Akroinfundibulum spielt nur selten eine wesentliche Rolle bei der Komedogenese. Das ist etwa bei der Teerakne, die zu den berufsbedingten Akneformen zählt, der Fall. Bei dieser Akne ist der klinisch sichtbare Komedotyp nicht der geschlossene, sondern der offene Komedo. Der obere Abschnitt des Infundibulums ist in diesem besonderen Fall also von Beginn an in das Geschehen mit einbezogen und erweitert sich mit Einsetzen der follikulären Hyperkeratose.

Die follikuläre Hyperkeratose beruht auf einer Veränderung in der Differenzierung der Keratinozyten. Die Keratinozyten liegen dicht nebeneinander, wobei die erhöhte Anzahl an Tonofilamenten und Desmosomen im Infrainfundibulum zu einer gesteigerten Kohäsion der Zellen führt. Die verminderte Anzahl der Keratinosomen (engl. „odland bodies“) bedingt Veränderungen im Lipidmetabolismus der Keratinozyten [62]. Darüber hinaus ist eine gesteigerte proliferative Zellaktivität nachweisbar.

Allerdings haben sich keine auffälligen Unterschiede in der Expression verschiedener desmosomaler Antigene bei Aknepatienten und Hautgesunden ergeben, die als Erklärung für die Kohärenz der Follikelkeratinozyten bei der Entstehung von Komedonen herangezogen werden könnten [28]. Andererseits findet sich im Zusammenhang mit der Entstehung der Akne eine vermehrte Expression von Tenascin, einem extrazellulären Matrixprotein, das mit Zellen interagieren und ihre Adhäsionskapazität beeinflussen kann [29].

Für die follikuläre Hyperkeratose werden in der Pathogenese der Akne verschiedene Ursachen angeschuldigt, auf die im folgenden Abschnitt eingegangen werden soll (Tab.1).

\section{Veränderungen der Lipidzusammensetzung}

Epidermale Lipide spielen eine wichtige Rolle bei der Aufrechterhaltung der Barrierefunktion und steigern die Wasserbindungskapazität. Die Konversion von Cholesterolsulfat zu Cholesterol, die unter dem Einfluss des Enzyms Steroidsulfatase erfolgt, stellt den entscheidenden Schritt in der Differenzierung der Keratinozyten dar. Eine genetisch bedingte verminderte Aktivität der Steroidsulfatase könnte daher zur Retentionshyperkeratose beitragen [37].

Es ist wahrscheinlich, dass Lipide die Proliferation und Differenzierung von Keratinozyten beeinflussen. $\mathrm{N}$-acylierte Formen der Sphingolipide, wie Ceramide, fördern die Keratinozytendifferenzierung, während Sphingosin und Sphingosylphosphorylcholin die Keratinozytenproliferation verstärken [58].

Mit der Vergrößerung der Talgdrüse soll es zu einer relativen Verminderung des Anteils der Linolsäure im Follikel kommen [9]. Die Bestimmung der polaren Lipide ergab, dass die Acylceramide aus Komedonen nur 6\% Linolsäure innerhalb der veresterten Fettsäuren im Vergleich zu $45 \%$ in den Acylceramiden der
Tab. 1 Mögliche endogene Ursachen der Proliferations-Retentions-Hyperkeratose

Veränderungen im Talgdrüsenfollikelzyklus

Androgene Effekte auf Follikelkeratinozyten

Veränderungen im Lipidmetabolismus (Squalen, freie Fettsäuren, Linolsäure)

Veränderungen des Retinol- und Dehydroretinolgehaltes der Talgdrüse

Cytochrom P-450-Polymorphismen

Mikrobielle Faktoren von P. acnes mit komedogenem Potential

Zytokine (insbesondere Interleukin- $1 \alpha$ )

normalen Epidermis enthielten [59]. Linolsäure ist eine essentielle Fettsäure, die von Sebozyten und Keratinozyten zum Aufbau von Membranlipiden benötigt wird. Zwischen der Linolsäurekonzentration im Talg und der Talgsekretionsrate besteht eine umgekehrt proportionale Beziehung [51]. Die Abnahme der Talgsekretionsrate unter systemischer Therapie mit Isotretinoin [36] oder Antiandrogenen [50] geht mit einem Anstieg sowohl der Linolsäurekonzentration in der Wachsesterfraktion der Talgdrüsenlipide als auch der Triglyzeride der freien Fettsäuren und der Cholesterinperoxidester in den Oberflächenlipiden einher. Mit dem verminderten Anteil der Linolsäure im Talg gelangt auch ein geringerer Anteil an die luminalen Keratinozyten des Infrainfundibulums, die hierdurch eine veränderte terminale Differenzierung zeigen. In Tierexperimenten konnte gezeigt werden, dass eine linolsäurearme Ernährung mit erhöhtem transepidermalen Wasserverlust als Ausdruck einer verminderten epidermalen Barrierefunktion und Hyperproliferation des Epithels (Hyperkeratose) einhergeht.

\section{Androgene}

Androgene steigern nicht nur die Größe und Aktivität der Talgdrüsen, sondern sind möglicherweise auch an der Ausbildung der follikulären Hyperkeratose beteiligt. Allerdings gibt es für diese Annahme bisher nur indirekte Belege. Androgenrezeptoren kommen in der äußeren Wurzelscheide des Infrainfundibulums des Follikels vor [5]. Die Ausbildung von Mikrokomedonen nimmt unter systemischer Therapie mit Antiandrogenen ab [6]. Die Schlüsselenzyme des Androgenmetabolismus wurden im Follikel nachgewiesen [16]. Die Aktivität des Enzyms Typ 1-5 $\alpha$-Reduktase, das Testosteron zu dem wirksameren Dihydrotestosteron konvertiert, ist in Follikelkeratinozyten stärker als in epidermalen Keratinozyten [52,53]. Follikelkeratinozyten scheinen demnach mehr als epidermale Keratinozyten zur Metabolisierung von Androgenen in der Lage zu sein.

\section{Veränderungen im Vitamin-A-Metabolismus}

Eine andere Erklärung für die Hyperkeratose des Follikelkanals besteht darin, dass durch die erhöhte Talgsekretionsrate im Follikel ein lokaler Mangel an Retinol oder Dehydroretinol entsteht [24]. Hierfür sprechen Befunde, die eine quantitative Verschiebung von endogenen Retinoiden unter systemischer Therapie mit Isotretinoin nachweisen konnten [56]. Ein Vitamin-A-Mangel (Hypovitaminose A) äußert sich klinisch als Verhornungsstörung. 
Vitamin A (Retinol) und andere endogene Retinoide antagonisieren den Effekt der Androgene auf die Talgdrüse und hemmen die Proliferation der Sebozyten. Bei einigen Aknepatienten wurde eine Mutation im Gen von Cytochrom P-450 1A1 gefunden [40]. Hieraus resultiert ein beschleunigter Abbau und damit ein Mangel an endogenen Retinoiden. Darin könnte eine weitere Ursache für die gestörte Differenzierung der Sebozyten und Hyperkeratose des Follikelkanals begründet sein.

\section{Zytokine}

Guy und Kealey $[14,15]$ untersuchten den Einfluss des Zytokins Interleukin- $1 \alpha$ auf die Verhornung des Follikels und Entstehung von Komedonen (Abb. 1). Interleukin-1 $\alpha$ ist ein pro-inflammatorisches Zytokin, das von Lymphozyten und Keratinozyten gebildet wird. Die Autoren wiesen Interleukin-1 $\alpha$ innerhalb der Komedonen und des Follikelepithels nach. Nach Zugabe von Interleukin-1 $\alpha$ zu kultivierten infrainfundibulären Keratinozyten trat eine follikuläre Verhornung ein. Die Ausbildung der Hyperkeratose konnte durch Zugabe von Interleukin-1-Rezeptor-Antagonist blockiert werden. Die Autoren ziehen den Schluss, dass Interleukin-1 $\alpha$, Interleukin-1-Rezeptor und Interleukin-1-Rezeptor-Antagonist zur Entstehung von Komedonen beitragen.

In vitro ließ sich die Interleukin- $1 \alpha$-induzierte Komedonenbildung durch Zugabe von epidermalem Wachstumsfaktor (EGF) oder Tumornekrosefaktor (TNF) $-\alpha$ hemmen $[14,15]$.

Interleukin- $1 \alpha$ ist in Follikeln mit hoher P. acnes-Kolonisierungsdichte nachweisbar [20,21]. P. acnes, ein im Infrainfundibulum kolonisierendes Gram-positives Bakterium, produziert in vitro Faktoren wie Histamin, Tryptamin und kurzkettige Fettsäuren [2]. Falls das auch in vivo der Fall ist, könnte P. acnes direkt zur Freisetzung von Interleukin- $1 \alpha$ beitragen.

Weiterhin muss Interleukin- $1 \alpha$ als Mediator der T-Zell-Aktivierung verstanden werden, der bei der Initiierung der entzündlichen Akne eine wichtige Rolle spielt.

\section{Propionibakterien}

Der in den Talgdrüsenfollikeln reichlich vorhandene Talg bietet $P$. acnes ein anaerobes, lipidreiches Mikromilieu, in dem sich das Bakterium vermehren kann. P. acnes produziert eine Vielzahl von extrazellulären Enzymen, darunter eine Lipase, die im Talg enthaltene Triglyzeride zu Glycerol, das als Wachstumssubstrat dient, und freien Fettsäuren mit pro-inflammatorischen und komedogenen Eigenschaften hydrolysiert [23,32,33].

Weiterhin könnte das Lipid Squalen in die Pathogenese der Akne involviert sein, da die Oxidation von Squalen im Follikelkanal unter dem Einfluss von P. acnes durch Reduzierung der lokalen Sauerstoffspannung ein geeignetes Mikromilieu für das Wachstum von $P$. acnes in diesem Follikelabschnitt schafft und das Oxidationsprodukt Squalenoxid die Komedogenese begünstigt [47] (Abb. 2). Die Oxidation von Squalen wird durch Porphyrine, die von $P$. acnes gebildet werden, nach Freisetzung in das Lumen katalysiert.

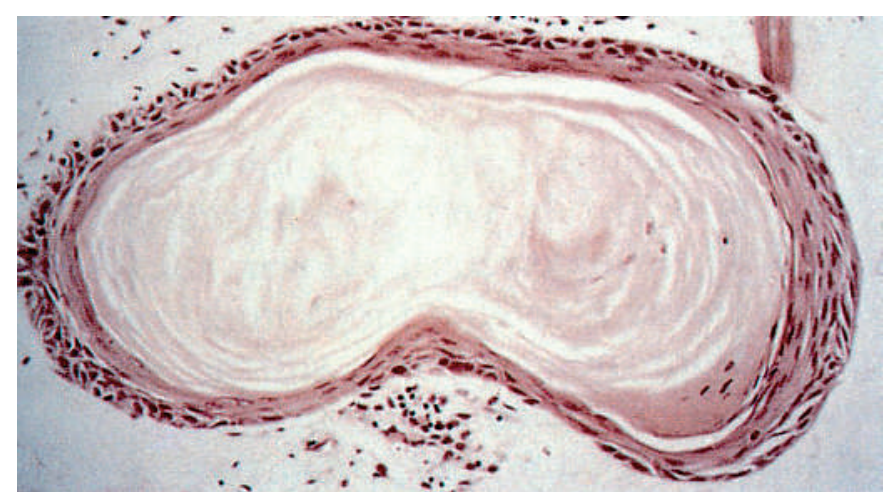

Abb. 1 In vitro durch Interleukin-1 $\alpha$ induzierter Komedo (Mit freundlicher Genehmigung von Dr. R. Guy, Cambridge, UK).
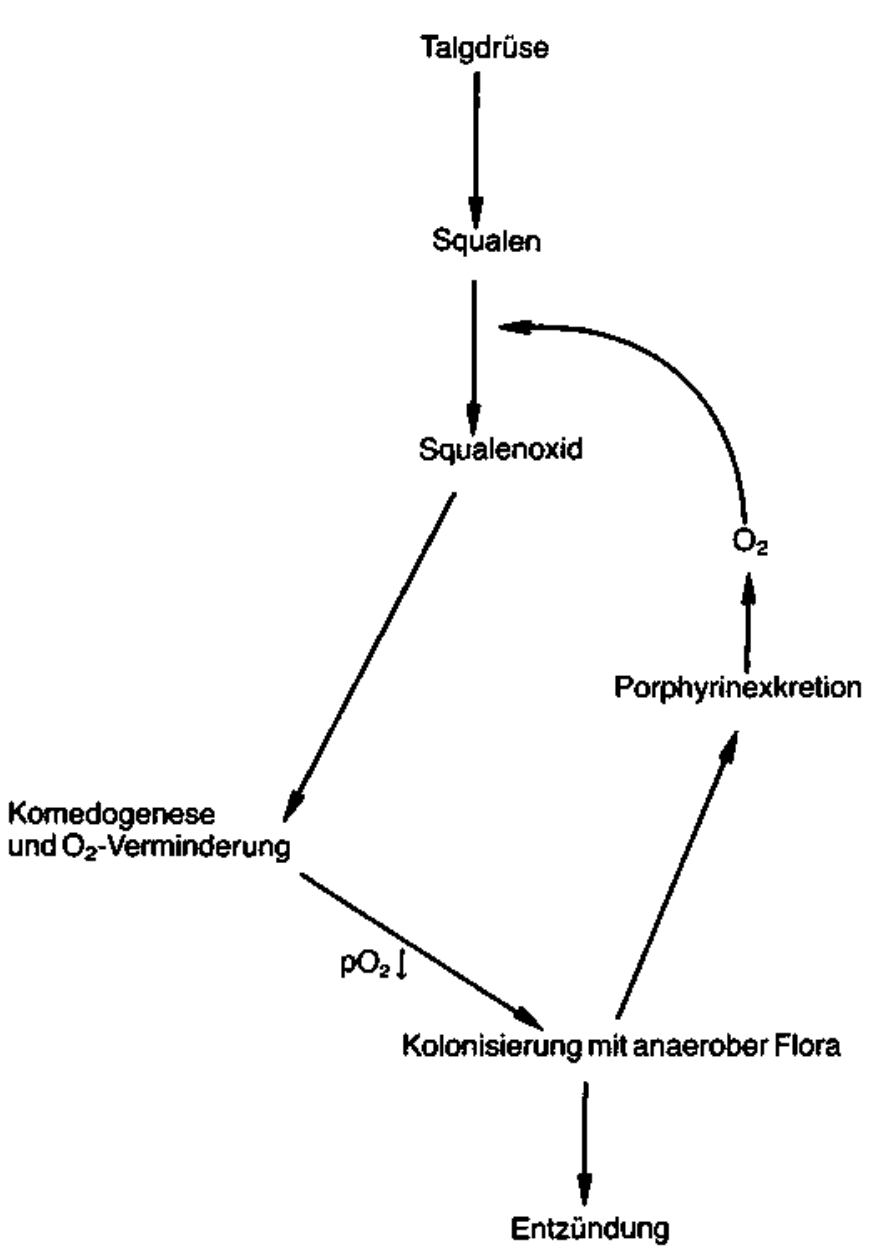

Abb. 2 Mögliche Bedeutung von Squalen für die Pathogenese der Akne nach Saint-Leger u. Mitarb. [47].

Die Komedogenität von UV-A-bestrahlten und unbestrahlten Substanzen (Squalen, Ölsäure, Isopropylmyristat, Squalenperoxid, flüssiges Paraffin) wurde im Kaninchenohrmodell mittels Oberflächenmikroskopie und histologisch untersucht [38]. Squalenperoxid erwies sich als hochgradig komedogen, dagegen Squalen selbst nur geringgradig. Sowohl Ölsäure als auch ihr Peroxid waren in der Lage, relativ große Komedonen zu induzieren, wobei die Konzentration der Lipidperoxide mit der Größe der Komedonen gut korrelierte. Wenn also Bestandteile des Talges an der Komedogenese beteiligt sind, so sind Squalen und freie 
Fettsäuren weniger komedogen als ihre Peroxide. Diese Untersuchungen unterstützen die Annahme, dass UV-A-Bestrahlung die Fähigkeit von Talg und Squalen, am Kaninchenohr komedogen zu wirken, erhöht. Allerdings lassen sich die Ergebnisse aus dem Tiermodell nicht ohne weiteres auf den Menschen übertragen, so dass die Bedeutung von Squalen und freien Fettsäuren für die Komedogenese spekulativ bleibt.

P. acnes ist nicht in jedem Follikel nachweisbar. Wenn Gewebeproben vor der Pubertät entnommen werden, finden sich Mikrokomedonen mit follikulärer Hyperkeratose ohne Präsenz von $P$. acnes [39]. Das bedeutet, dass die mikrobielle Hyperkolonisation des Follikels mit $P$. acnes keine notwendige Voraussetzung für die Entstehung von Komedonen darstellt.

Es gibt Hinweise darauf, dass Sebozyten in Abwesenheit von $P$. acnes in der Lage sind, Interleukin- $1 \alpha$ und freie Fettsäuren $\mathrm{zu}$ produzieren $[12,63]$. Sebozyten könnten daher als zusätzliche intrinsische Faktoren zur Entstehung von entzündlicher und nicht-entzündlicher Akne beitragen.

\section{Exogene Faktoren}

Nicht unerwähnt bleiben sollen exogene Faktoren, die zur Komedogenese beitragen. In erster Linie zu nennen sind komedogene Substanzen, die in manchen Pflegemitteln und Kosmetika enthalten sind [13]. Hierzu zählen Fettsäureester (z. B. Butylstearat, Isopropylmyristat), Fettsäuren (z. B. Stearinsäuren) und Fettalkohole. Diese können bei längerer Anwendung eine geringfügige Komedonenakne provozieren. Dicht stehende offene Komedonen an Kinn und Wangen bestimmen das klinische Erscheinungsbild der Kontaktakne (Acne venenata), während geschlossene Komedonen selten und kaum zu finden sind. Beispiele sind die Kosmetik- und Pomadenakne [26,44]. Die ausgedehntesten und schwersten Fälle von Kontaktakne kommen in der Industrie im Rahmen von berufsbedingter Exposition vor, wobei zu den nicht-entzündlichen oftmals entzündliche Effloreszenzen hinzutreten. Beispiele sind die Öl-, Teer-, Pech- und Chlorakne [55]. Als Besonderheit wirken bei der Chlorakne die komedogenen Substanzen, in der Regel halogenierte Kohlenwasserstoffe, zwar überwiegend direkt über die Haut, seltener aber auch indirekt über orale oder pulmonale Ingestion ein.

Die Komedogenität wird üblicherweise im Kaninchenohrmodell geprüft, wobei die Komedonenbildung direkt proportional der komedogenen Potenz der getesteten Substanz ist [11].

Als weitere exogene Faktoren sind Medikamente zu nennen. So können etwa topisch oder systemisch applizierte Glukokortikoide zu auffällig monomorphen, follikulär gebundenen Papeln und Pusteln führen, die sich später in Komedonen umwandeln [19]. Der Komedo folgt der Entzündung, daher ist die Sequenz bei der Steroidakne umgekehrt wie bei der Acne vulgaris.

Es wird angenommen, dass die Keratinozyten im Follikelkanal in ähnlicher Weise wie die Keratinozyten an der Hautoberfläche auf eine verstärkte Hydration des Stratum corneum reagieren $[8,60]$. Eine Verlegung des Follikelkanals als Folge von starker Hydration, etwa beim Schwitzen, führt zu einer vorübergehen- den Blockierung der Talgsekretion. Unter bestimmten Bedingungen, etwa bei der Acne tropicalis, begünstigt eine starke Hydration die Entstehung der Akne [31]. Die Erfahrungen mit dieser Akneform gehen überwiegend auf Beobachtungen in den Tropen bei Kampfverbänden im Zweiten Weltkrieg sowie im Korea- und Vietnamkrieg zurück. Eine Besserung des schweren Krankheitsverlaufes gelang nur durch Rückkehr der Patienten in ein gemäßigtes trockenes Klima. Diese Beobachtungen zeigen, dass Hitze und hohe Luftfeuchtigkeit die Entstehung der Akne begünstigen.

Komedonen treten auch als Folge von chronisch-aktinischer Belastung (aktinische Komedonen: Morbus Favre-Racouchot) [41] oder ionisierenden Strahlen (Kobalt oder Röntgen) [4] auf. Die zugrunde liegenden Mechanismen sind weitgehend unbekannt, allerdings könnte eine strahleninduzierte Metaplasie des Follikelepithels von Bedeutung sein. Als Folge werden die Keratinozyten nicht abgestoßen, sondern als Komedo retiniert.

\section{Offene Fragen}

Das Verständnis der Pathogenese der Akne hat in den letzten Jahren erhebliche Fortschritte erfahren, doch fehlen Antworten auf wichtige Fragen. So bleibt derzeit unklar, warum mit der Rückbildung der Komedogenese und Follikelhyperkeratose nicht auch die Talgdrüsenhyperplasie und Seborrhö zurückgehen, obwohl die Akne gewöhnlich durch die Rückbildung der Follikelhyperkeratose eine natürliche Regression erfährt.

\section{Pathogenese-orientierte Aknetherapie}

Die Normalisierung der gestörten Verhornung in den Ausführungsgängen der Talgdrüsenfollikel ist das primäre Therapieziel bei nicht-entzündlicher Akne. Zur Lokaltherapie der Acne comedonica sind in erster Linie Retinoide, Azelainsäure, Benzoylperoxid, Salizylsäure und Alpha-Hydroxy-Säuren geeignet, wobei sich Retinoide durch eine überlegene komedolytische Wirksamkeit auszeichnen.

$\mathrm{Zu}$ den Retinoiden zählen Tretinoin (all-trans-Retinsäure, Vitamin-A-Säure), Isotretinoin (13-cis-Retinsäure), Adapalen und Tazaroten. Mit Tazaroten liegen positive Erfahrungen in der Aknetherapie vor, der Wirkstoff ist in Deutschland jedoch nicht für diese Indikation zugelassen [3].

Retinoide stellen die normale epitheliale Keratinisierung wieder her, wirken der Neubildung von Mikrokomedonen entgegen, fördern die Abheilung von Komedonen und sind gegen Papulopusteln wirksam. Darüber hinaus beeinflussen sie indirekt durch Veränderung des follikulären Mikromilieus das Wachstum von P. acnes.

Im folgenden Abschnitt soll auf die molekularen Wirkungsmechanismen der Retinoide und exemplarisch auf die Bedeutung des Rezeptor-selektiven Retinoids Adapalen als Aknetherapeutikum eingegangen werden.

Topisch applizierte Retinoide wirken in der Zelle durch Interaktion mit spezifischen Retinsäure-Rezeptoren, $\operatorname{RAR}(\alpha, \beta, \gamma)$, und Re- 
tinoid-X-Rezeptoren, RXR ( $\alpha, \beta, \gamma)$ [46] (Tab. 2). Auf diese Weise beeinflussen sie die Expression von Genen, die für die Zellproliferation und -differenzierung sowie Entzündungsreaktion verantwortlich sind. Die nukleären Retinoidrezeptoren gehören wie die Rezeptoren für Vitamin D, Estradiol, Glukokortikoide und Thyroidhormone zur Großfamilie der nukleären Hormonrezeptoren, die als Liganden-abhängige Transkriptionsfaktoren agieren. Sie verbinden sich mit den Retinoiden nicht als Einzelmoleküle, sondern in Form von Dimeren, als Homodimere (RAR/RAR, RXR/ RXR) oder Heterodimere (RAR/RXR). Bei den Heterodimeren übernimmt RXR und nicht RAR die Genregulation und gilt damit als Superrezeptor.

Die Retinoidrezeptoren zeigen unterschiedliche Affinitäten für die verschiedenen Retinoide und eine charakteristische Gewebeverteilung. RAR- $\alpha$ kommt in der Epidermis nur im Stratum germinativum vor. RAR- $\beta$ findet sich hauptsächlich in der Dermis, wird aber auch in anderen Körpergeweben exprimiert. RAR- $\gamma$ wird vorwiegend in der Epidermis gefunden. RAR- $\alpha$ wird mit Proliferation der Keratinozyten und RAR- $\gamma$ mit terminaler Differenzierung der Keratinozyten in Verbindung gebracht.

Der Retinsäure-Rezeptor-Dimer-Komplex aktiviert bestimmte Gene, die ein Retinsäure-Response-Element (RARE) besitzen. Dadurch kommt es zur Transkription (mRNA-Synthese) dieser Gene und Translation (Proteinsynthese) und damit zum Retinoideffekt. Das zytosolische Retinsäure-bindende Protein (CRABP), das während des Verhornungsprozesses der epidermalen Keratinozyten unterschiedlich exprimiert wird, reguliert die Konzentration der Retinsäure in den Zellen und daher indirekt auch die Genexpression.

Tretinoin wird durch das CRABP vom Zytoplasma zum Kern transportiert, wo ein Komplex mit einem Rezeptorprotein aufgebaut wird. Dort interagiert es mit allen RAR-Rezeptoren, nicht jedoch mit den RXR-Rezeptoren, während Isotretinoin, das Stereoisomer von Tretinoin, an keinen der bekannten RAR- und RXR-Rezeptoren bindet und fraglich mit dem CRABP interagiert. Nach topischer Applikation wird Isotretinoin durch UV-Strahlung teilweise zu Tretinoin isomerisiert. Isotretinoin ist hinsichtlich des Wirkeffektes mit Tretinoin vergleichbar, allerdings hat Isotretinoin eine stärkere anti-inflammatorische Wirkung und führt zu einer geringeren lokalen Hautirritation. Im Unterschied zu der systemischen Isotretinointherapie wird die Talgsekretion durch topisch appliziertes Isotretinoin nicht beeinflusst.

Tretinoin, das über Jahrzehnte als Goldstandard für topisch applizierte Retinoide galt, greift früh in die Zytokeratinbildung ein. Dies äußert sich in einer erhöhten Desquamation und ausgeprägten Umstrukturierung der Epidermis und des follikulären Epithels. Der Einsatz von Tretinoin in der Aknetherapie ist durch die Häufigkeit und Schwere der lokalen Nebeneffekte bis hin zur Exazerbation der Akne in der Initialphase der Therapie limitiert.

Adapalen, ein neuartiges synthetisches Derivat der Naphthoesäure, ist zwar kein Retinoid im ursprünglichen Sinne, hat aber Retinoid-ähnliche Eigenschaften [49]. Der Wirkstoff interagiert selektiv mit dem RAR- $\beta$ - und RAR- $\gamma$-Rezeptor, nicht jedoch mit dem RAR- $\alpha$-Rezeptor, RXR-Rezeptor und dem CRABP. Tazaroten, ein synthetisches, nicht isomerisierbares, azetyliertes Retinoid,
Tab. 2 Retinoidrezeptor-Affinität von topisch applizierten Retinoiden

\begin{tabular}{llllllll} 
& CRABP & \multicolumn{3}{c}{ RAR } & \multicolumn{3}{c}{ RXR } \\
& & $\alpha$ & $\beta$ & $\gamma$ & $\alpha$ & $\beta$ & $\gamma$ \\
\hline Tretinoin & + & + & + & + & - & - & - \\
\hline Isotretinoin* & $+(?)$ & - & - & - & - & - & - \\
\hline Adapalen & - & - & + & + & - & - & - \\
\hline Tazaroten** & - & - & + & + & - & - & -
\end{tabular}

CRABP $=$ Zytosolisches Retinsäure-bindendes Protein, RAR = Retinsäure-Rezeptor, RXR = Retinoid-X-Rezeptor

* wird teilweise zu Tretinoin isomerisiert

** über Tazarotensäure

hat ein ähnliches Rezeptorprofil wie Adapalen. Da sich die Akne im wesentlichen als eine Differenzierungsstörung der Keratinozyten im Bereich des Talgdrüseninfundibulums auffassen lässt, könnte die Rezeptorselektivität von Retinoiden zu klinischen Vorteilen führen.

Die Modifikation der molekularen Struktur von Tretinoin, die zur Synthese von Adapalen geführt hat, bedingt noch weitere Fortschritte. So gelang es durch den Ersatz der in den Vitamin-A-Derivaten vorhandenen empfindlichen Doppelbindungen durch aromatische Ringe im Adapalenmolekül, das eine polyaromatische Verbindung darstellt, sowohl die chemische Instabilität als auch die Photoinstabilität früherer Retinoide zu überwinden.

Adapalen zeichnet sich dadurch aus, dass es eine geringe perkutane Absorption aufweist, wenn der Wirkstoff das Stratum corneum passiert hat. Adapalen interagiert hauptsächlich mit epidermalen Keratinozyten, deren Verhornungsmuster sich durch Hemmung der Keratinozyten-Transglutaminase ändert und deren Proliferationsrate abnimmt. Im Kern bindet Adapalen selektiv an den RAR- $\beta$ - und RAR- $\gamma$-Rezeptor. Dieser Komplex interagiert mit der DNA und aktiviert spezifische, bisher nicht identifizierte Gene.

Die Untersuchung von Follikelfilamenten mit Zyanoakrylattechnik ergab eine signifikante Zunahme der Ceramidkonzentration unter Therapie mit Adapalen oder Tretinoin [54]. Diese Befunde lassen darauf schließen, dass diese Wirkstoffe die follikuläre Keratinisierung beeinflussen und die epidermale Barrierefunktion stabilisieren.

Darüber hinaus konnte in mehreren Studien eine intrinsische anti-inflammatorische Aktivität von Adapalen nachgewiesen werden, die auf einer Hemmung des oxidativen Metabolismus der Arachidonsäure über den Lipoxygenase-Signaltransduktionsweg beruht, während sich der Effekt auf die Cyclooxygenase gering zeigte. In Entzündungsmodellen erwies sich Adapalen als ebenso wirksam wie Indometacin und Betamethason-17-valerat. Diese Eigenschaften könnten dazu beitragen, dass Adapalen im Vergleich zu anderen Retinoiden weniger Erytheme und andere Lokalreaktionen hervorruft. Daher ist Adapalen, in Kombination mit anderen Aknetherapeutika, als Retinoid der ersten Wahl in der Therapie von Aknepatienten mit atopischer oder empfindlicher Haut anzusehen. 
Eine weitere Erklärung für die anti-inflammatorische Wirksamkeit von Adapalen auf Zellebene besteht in der Hemmung der Expression von Toll-like-Rezeptoren (TLR). In vitro konnte gezeigt werden, dass Adapalen die Expression von TLR-2 auf Monozyten und Makrophagen hemmt, so dass die Zytokinfreisetzung aus diesen Zellen und damit die Entzündungsreaktion verhindert wird [57].

Zusätzlich beeinflusst Adapalen indirekt den Transkriptionsfaktor Aktivatorprotein (AP)-1, der sich aus den c-Jun- und c-FosGenprodukten zusammensetzt. AP-1 wird eine wesentliche Bedeutung in Hinblick auf Zellwachstum und Zellproliferation zugesprochen. Dies stellt einen weiteren Signaltransduktionsweg dar, über den der Wirkstoff indirekt zelluläre Funktionen auf Rezeptorebene beeinflussen kann.

Azelainsäure ist eine gesättigte, geradkettige $C_{9}$-Dikarbonsäure mit komedolytischen und antimikrobiellen Eigenschaften [10]. Die komedolytische Wirkung, die im Vergleich zu Retinoiden schwächer ausgeprägt ist, beruht auf einem Effekt auf die terminale Differenzierung der Keratinozyten. Damit nimmt Azelainsäure im Vergleich zu Tretinoin Einfluss auf spätere Schritte der Differenzierung. Da Azelainsäure die Melaninsynthese der Melanozyten hemmt, bietet sich die Substanz auch zur Therapie von postinflammatorischen Hyperpigmentierungen an. Die Substanz ist toxikologisch unbedenklich und daher auch in der Schwangerschaft einsetzbar. Aufgrund der guten lokalen Verträglichkeit wird sie von Patienten mit atopischer oder empfindlicher Haut bevorzugt.

Andere keratolytische Wirkstoffe wie Benzoylperoxid, Salizylsäure und Alpha-Hydroxy-Säuren haben nur einen geringen Einfluss auf die follikuläre Hyperkeratose und spielen daher nur eine untergeordnete Rolle in der Therapie der Komedonenakne.

Da der Akne eine multifaktorielle Genese zugrunde liegt, sollte die komedolytische Therapie mit anderen (antimikrobiellen, anti-inflammatorischen, sebosuppressiven) Therapieformen kombiniert werden, um möglichst viele pathogenetische Faktoren zu beeinflussen [35]. Klinische Studien haben gezeigt, dass die Kombination von topisch applizierten Retinoiden mit topisch oder systemisch applizierten Antibiotika oder anderen antimikrobiellen Substanzen in dieser Hinsicht besonders gut geeignet ist. Die Kombination von Retinoiden und antimikrobiellen Substanzen führt zu einer rascheren und stärkeren Reduzierung der Akneeffloreszenzen als die Applikation der Einzelsubstanzen. Daher verbessert diese Kombinationstherapie sowohl die Compliance der Aknepatienten als auch deren Selbstbewusstsein und Wohlbefinden.

Die Vorteile der Kombinationstherapie wirken sich besonders dann aus, wenn die Einzelsubstanzen von Beginn an kombiniert eingesetzt werden. Sobald eine wesentliche Reduzierung der Akneeffloreszenzen oder entscheidende klinische Besserung der Akne erkennbar ist, werden die antimikrobiellen Substanzen abgesetzt und die Therapie mit Retinoiden oder Azelainsäure fortgesetzt. Die Erhaltungstherapie ist erforderlich, um der Entstehung von neuen Mikrokomedonen entgegen zu wirken.
Um den komedolytischen Effekt zu optimieren, wird die Pharmakotherapie der Akne mit einer manuellen Komedonenextraktion kombiniert, mit deren Hilfe geschlossene in offene Komedonen transformiert und offene Komedonen beseitigt werden [48]. Die Komedonenextraktion hat zwar keinen Einfluss auf den Verlauf der Akne, jedoch hat sie in der Initialphase der Therapie einen Compliance-fördernden Effekt.

\section{Literatur}

${ }^{1}$ Aldana OL, Holland DB, Cunliffe WJ. Variation in pilosebaceous duct keratinocyte proliferation in acne patients. Dermatology 1998; 196: 98-99

${ }^{2}$ Allaker RP, Greenman J, Osborne RH. The production of inflammatory compounds by Propionibacterium acnes and other skin organisms. $\mathrm{Br} \mathrm{J}$ Dermatol 1987; 117: $175-183$

${ }^{3}$ Anderson DN, Callender VD, Del Rosso JQ Tanghetti EA, Zane LT. Topical tazarotene in acne vulgaris: treatment approaches. Cutis 2004; 74 (Suppl 4): 16-21

${ }^{4}$ Aversa AJ, Nagy R. Localized comedones following radiation therapy. Cutis 1983; 31: 296-303

${ }^{5}$ Choudhry R, Hodgkins MB, Van der Kwast TH, Brinkmann AO, Boersma WJ. Localization of androgen receptors in human skin by immunocytochemistry: implications for the hormonal regulation of hair growth, sebaceous glands and sweat glands. J Endocrinol 1992; 133: $467-475$

${ }^{6}$ Cunliffe WJ, Forster RA. Androgen control of the pilosebaceous duct? Br J Dermatol 1987; 116: 449

${ }^{7}$ Cunliffe WJ, Holland DB, Clark SM, Stables GI. Comedogenesis: some new aetiological, clinical and therapeutic strategies. Br J Dermatol 2000; 142: $1084-1091$

${ }^{8}$ Cunliffe WJ, Perera WD, Tan SG, Williams M, Williams S. Pilo-sebaceous duct physiology. II. The effect of keratin hydration on sebum excretion rate. $\mathrm{Br}$ J Dermatol 1976; 94: 431 -434

${ }^{9}$ Downing DT, Stewart ME, Wertz PW, Strauss JS. Essential fatty acids and acne. J Am Acad Dermatol 1986; 14: 221 -225

${ }^{10}$ Fitton A, Goa KL. Azelaic acid. A review of its pharmacological properties and therapeutic efficacy in acne and hyperpigmentary skin disorders. Drugs 1991; 41: 780-798

${ }^{11}$ Frank SB. Is the rabbit ear, in its present state, prophetic of acnegenicity? J Am Acad Dermatol 1982; 6: 373-377

${ }^{12}$ Fujie T, Shikiji T, Uchida N, Urano Y, Nagae H, Arase S. Culture of cells derived from the human sebaceous gland under serum-free conditions without a biological feeder layer or specific matrices. Arch Dermatol Res 1996; 288: $703-708$

${ }^{13}$ Fulton JE Jr. Comedogenicity and irritancy of commonly used ingredients in skin care products. J Soc Cosmet Chem 1989; 40: 321 - 333

${ }^{14}$ Guy R, Kealey T. Modelling the infundibulum in acne. Dermatology 1998; 196: $32-37$

15 Guy R, Kealey T. The effects of inflammatory cytokines on the isolated human sebaceous infundibulum. J Invest Dermatol 1998; 110: 410415

${ }^{16}$ Hay JB, Hodgins MB. Distribution of androgen metabolizing enzymes in isolated tissues of human forehead and axillary skin. J Endocrinol 1978; 79: 29-39

17 Holland DB, Aldana OL, Cunliffe WJ. Abnormal integrin expression in acne. J Invest Dermatol 1998; 110: 559 (Abstract)

${ }^{18}$ Hughes BR, Morris C, Cunliffe WJ, Leigh IM. Keratin expression in pilosebaceous epithelia in truncal skin of acne patients. Br J Dermatol 1996; 134: 247-256

${ }^{19}$ Hurwitz RM. Steroid acne. J Am Acad Dermatol 1989; 21: 1179-1181

${ }^{20}$ Ingham E, Eady EA, Goodwin CE, Cove JH, Cunliffe WJ. Pro-inflammatory levels of interleukin- $1 \alpha$-like bioactivity are present in the majority of open comedones in acne vulgaris. J Invest Dermatol 1992; 98: 895-901

${ }^{21}$ Ingham E, Walters CE, Eady EA, Cove JH, Kearney JN, Cunliffe WJ. Inflammation in acne vulgaris: failure of skin micro-organisms to modulate keratinocyte interleukin $1 \alpha$ production in vitro. Dermatology 1998; 196: 86-88

${ }^{22}$ Jansen T, Plewig G, Kligman AM. Pathophysiology of acne. Dermatol Ther 1998; 6: 7-17 
${ }^{23}$ Kanaar P. Follicular-keratogenic properties of fatty acids in the external ear canal of the rabbit. Dermatologica 1971; 142: 14-22

${ }^{24}$ Kealey T. Hypovitaminosis A of follicular duct as cause of acne vulgaris. Lancet 1988; II: 449

${ }^{25}$ Kligman AM. An overview of acne. J Invest Dermatol 1974; 62: 268 287

${ }^{26}$ Kligman AM. A critical look at acne cosmetica. J Cutan Aging Cosmet Dermatol 1988/89; 1: 109-114

${ }^{27}$ Knaggs HE, Holland DB, Morris C, Wood EJ, Cunliffe WJ. Quantification of cellular proliferation in acne using the monoclonal antibody Ki-67. J Invest Dermatol 1994; 102: 89-92

${ }^{28}$ Knaggs HE, Hughes BR, Morris C, Wood EJ, Holland DB, Cunliffe WJ. Immunohistochemical study of desmosomes in acne vulgaris. $\mathrm{Br} J$ Dermatol 1994; 130: 731 - 737

${ }^{29}$ Knaggs HE, Layton AM, Morris C, Wood EJ, Holland DB, Cunliffe WJ. Investigation of the expression of the extracellular matrix glycoproteins tenascin and fibronectin during acne vulgaris. Br J Dermatol 1994; 130: $576-582$

${ }^{30}$ Knutson D. Ultrastructural observations in acne vulgaris: the normal sebaceous follicle and acne lesions. J Invest Dermatol 1974; 62: 288 307

${ }^{31}$ Lamberg SI. The course of acne vulgaris in military personnel stationed in Southeast Asia. Cutis 1971; 7: 655-660

${ }^{32}$ Lavker RM, Leyden JJ, McGinley KJ. The relationship between bacteria and the abnormal follicular keratinization in acne vulgaris. J Invest Dermatol 1981; 77: 25-330

${ }^{33}$ Leeming JP, Holland KT, Cunliffe WJ. The pathological and ecological significance of microorganisms colonising acne vulgaris comedones. J Med Microbiol 1985; 20: 11-16

${ }^{34}$ Leyden JJ. New understandings of the pathogenesis of acne. J Am Acad Dermatol 1995; 32: S15-S25

${ }^{35}$ Leyden JJ. A review of the use of combination therapies for the treatment of acne vulgaris. J Am Acad Dermatol 2003; 49 (Suppl 3): S200 S210

${ }^{36}$ Melnik B, Kinner T, Plewig G. Influence of oral isotretinoin treatment on the composition of comedonal lipids: implications for comedogenesis in acne vulgaris. Arch Dermatol Res 1988; 280: 97- 102

37 Milewich L, Sontheimer RD, Herndon JH Jr. Steroid sulfatase activity in epidermis of acne-prone and non-acne-prone skin of patients with acne vulgaris. Arch Dermatol 1990; 126: 1312-1314

${ }^{38}$ Motoyoshi K. Enhanced comedo formation in rabbit ear skin by squalene and oleic acid peroxides. Br J Dermatol 1983; 109: 191 - 198

${ }^{39}$ Norris JF, Cunliffe WJ. A histological and immunocytochemical study of early acne lesions. Br J Dermatol 1988; 118: 651 -659

40 Paraskevaidis A, Drakoulis N, Roots I, Orfanos CE, Zouboulis CC. Polymorphisms in the human cytochrome P-450 1A1 gene (CYP1A1) as a factor for developing acne. Dermatology 1998; 196: 171 - 175

${ }^{41}$ Patterson WM, Fox MD, Schwartz RA. Favre-Racouchot disease. Int J Dermatol 2004; 43: 167-169

${ }^{42}$ Pawin H, Beylot C, Chivot M, Faure M, Poli F, Revuz J, Dréno B. Physiopathology of acne vulgaris: recent data, new understanding of the treatments. Eur J Dermatol 2004; 14: 4-12

${ }^{43}$ Plewig G. Folicular keratinization. J Invest Dermatol 1974; 62: 308 320

${ }^{44}$ Plewig G, Fulton JE Jr, Kligman AM. Pomade acne. Arch Dermatol 1970; 101: 580

45 Plewig G, Fulton JE Jr, Kligman AM. Cellular dynamics of comedo formation in acne vulgaris. Arch Dermatol Forsch 1971; 242: 12-29
${ }^{46}$ Rigopoulos D, Ioannides D, Kalogeromitros D, Katsambas AD. Comparison of topical retinoids in the treatment of acne. Clin Dermatol 2004; 22: $408-411$

${ }^{47}$ Saint-Leger D, Bague A, Lefebvre E, Cohen E, Chivot M. A possible role for squalene in the pathogenesis of acne. II. In vivo study of squalene oxides in skin surface and intra-comedonal lipids of acne patients. $\mathrm{Br} \mathrm{J}$ Dermatol 1986; 114: $543-552$

${ }^{48}$ Shalita AR, Harris H. Comedo extraction. Arch Dermatol 1972; 105 : $759-760$

${ }^{49}$ Shroot B, Michel S. Pharmacology and chemistry of adapalene. J Am Acad Dermatol 1997; 36 (Suppl 6): S96-S103

50 Stewart ME, Greenwood R, Cunliffe WJ, Strauss JS, Downing DT. Effect of cyproterone acetate-ethinyl estradiol treatment on the proportions of linoleic and sebaleic acids in various skin surface lipid classes. Arch Dermatol Res 1986; 278: 481 - 485

${ }^{51}$ Stewart ME, Wertz PW, Crahek MO. Relationship between sebum secretion rates and the concentration of linoleate in sebum and epidermal lipids. Clin Res 1985; 33: 684-688

52 Thiboutot DM, Knaggs H, Gilliland K, Hagari S. Acitivity of type 1 $5 \alpha$-reductase is greater in the follicular infrainfundibulum compared with the epidermis. Br J Dermatol 1997; 136: 166-171

${ }^{53}$ Thiboutot DM, Knaggs H, Gilliland K, Lin G. Activity of 5-alpha-reductase and 17-beta-hydroxysteroid dehydrogenase in the infundibulum of subjects with and without acne vulgaris. Dermatology 1998; 196: $38-42$

54 Thielitz A, Helmdach M, Röpke EM, Gollnick H. Lipid analysis of follicular casts from cyanoacrylate strips as a new method for studying therapeutic effects of antiacne agents. Br J Dermatol 2001; 145: 19-27

55 Tindall JP. Chloracne and chloracnegens. J Am Acad Dermatol 1985; 13: $539-558$

${ }^{56}$ Vahlquist A, Rollman O, Holland DB, Cunliffe WJ. Isotretinoin treatment of severe acne affects the endogenous concentration of vitamin A in sebaceous glands. J Invest Dermatol 1990; 94: 496-498

57 Vega B, Jomard A, Michel S. Regulation of human monocyte Toll-like receptor 2 (TLR2) expression by adapalene. J Eur Acad Dermatol Venereol 2002; 16: 123 - 124 (Abstract)

${ }^{58}$ Wakita H, Matsushita K, Nishimura K, Tokura Y, Furukawa F, Takigawa M. Sphingosylphosphorylcholine stimulates proliferation and upregulates cell surface-associated plasminogen activator activity in cultured human keratinocytes. J Invest Dermatol 1998; 110: 253-258

${ }^{59}$ Wertz PW, Miethke MC, Long SA, Strauss JS, Downing DT. The composition of the ceramides from human stratum corneum and from comedones. J Invest Dermatol 1985; 84: 410-412

${ }^{60}$ Williams M, Cunliffe WJ, Gould D. Pilo-sebaceous duct physiology. I. Effect of hydration on pilo-sebaceous duct orifice. Br J Dermatol 1974; 90: $631-635$

${ }^{61}$ Wolff HH, Plewig G, Braun-Falco O. Ultrastructure of human sebaceous follicles and comedones following treatment with vitamin A acid. Acta Derm Venereol (Stockh) 1975; 74 (Suppl): 99-100

62 Woo-Sam PC. A quantitative study of membrane coating granules in follicles undergoing experimental comedo formation. $\mathrm{Br}$ J Dermatol 1978; 99: $387-394$

${ }^{63}$ Zouboulis CC, Xia L, Akamatsu H, Seltmann H, Fritsch M, Hornemann S, Ruhl R, Chen W, Nau H, Orfanos CE. The human sebocyte culture model provides new insights into development and management of seborrhoea and acne. Dermatology 1998; 196: 21 - 31 